\title{
Análisis de la Diversidad de la Avifauna en tres Parques Urbanos de Guayaquil
}

\author{
Avifauna Diversity Analysis in three Guayaquil Urban \\ Parks
}

Thalia Zambrano Jaime ${ }^{1, *}$, Paola Peñafiel Villarreal ${ }^{1}$, Oswaldo Quiñonez Loor ${ }^{2}$

INFORMACIÓN DEL

ARTÍCULO

Fecha de recepción: 21 de noviembre de 2019.

Fecha de aceptación: 29 de enero de 2020 .

1 Universidad Agraria del Ecuador, Facultad de Ciencias agrarias, Guayaquil, Ecuador.

2 Universidad Técnica Estatal de Quevedo, Quevedo, Ecuador.

* Autor de correspondencia:

Thalia Zambrano Jaime. Universidad Agraria del Ecuador, Facultad de Ciencias Agrarias, Guayaquil, Ecuador.

E-mail: z.thaly18@gmail.com. Tlf: (+593) 989873341.

ENLACE DOI:

http://dx.doi.org/10.31095/investigatio. 2020.13 .3

\begin{abstract}
Resumen
Los parques urbanos son sitios de refugio, alimentación y anidación para las aves, por lo que son áreas prioritarias para su conservación. Se analizó la riqueza, abundancia y diversidad de la avifauna en los parques Centenario, Seminario y San Agustín, a través de la comparación de variables como la superficie de los parques y la distribución de aves en estratos verticales de la vegetación, para el establecimiento de puntos de conservación. Se empleó la metodología de puntos de conteos y se identificaron las aves utilizando la guía de campo Aves del Ecuador. Se determinó que el parque Centenario obtuvo mayor riqueza al registrar un total de 20 aves observadas; el parque Seminario, 17 aves; y el parque San Agustín, siete aves. Se registró tres especies globalmente amenazadas: Aratinga erythrogenys, Brotogeris pyrrhopterus y Conopias albovittata.
\end{abstract}

\section{Palabras Clave:}

Aves; diversidad; estratos de vegetación; Guayaquil; parques urbanos; riqueza.

\section{Clasificación JEL: Q57.}

\begin{abstract}
Urban parks are birds' refuge, feeding and nesting places. They are, therefore, priority conservation areas. The avifauna richness, abundance and diversity in Centenario, Seminario and San Agustin parks were analyzed through the comparison of variables such as park surface area and bird distribution in vertical vegetation strata for conservation points establishment. Counting point methodology was used and birds were identified using the Aves del Ecuador field guide (Birds of Ecuador). It was determined that Centenario Park showed greater richness, since it registered a total of 20 birds observed; Seminario Park, 17 bird; and San Agustin Park, 7 birds. Three globally threatened species were recorded: Aratinga erythrogenys, Brotogeris pyrrhopterus and Conopias albovittata.
\end{abstract}

\section{Keywords:}

Birds; diversity; vegetation strata; Guayaquil; urban parks; richness.

JEL Classification: Q57.

25

INVESTIGATIO No. 13, marzo 2020, pp. 25-40,

ISSN: 1390 - 6399・ISSN-e: 2602 - 8336 


\section{Introducción}

Ecuador tiene una riqueza inigualable de aves, el cual se han registrado a nivel nacional 1.685 especies (Freile et al., 2018; Ministerio del ambiente del Ecuador [MAE], 2015), ubicando al país en el tercer puesto a escala mundial con más especies de aves observadas en el marco del Global Big Day (eBird, 2019). Las razones que explican la diversidad en el país es la combinación de factores climáticos, biogeográficos, ecológicos y evolutivos (Burneo, 2009). Sin embargo, la Unión Internacional para la Conservación de la Naturaleza, ha registrado el $0,06 \%$ de las especies de aves extintas; el $6,65 \%$ se encuentran dentro de las categorías amenazadas; el 93\% están dentro de las categorías casi amenazadas y de preocupación menor y el $0,29 \%$ son aves poco estudiadas (BirdLife, 2018; IUCN, 2018).

La provincia del Guayas consta de un registro de 570 aves, representando el $73,86 \%$ de especies Nativas; 8,07\% especies endémicas de la región Tumbesina $y$ el $18,07 \%$ pertenecen a las especies endémicas de la Región Chocó, ladera del Sur, Ladera del Norte y especies introducidas (Prefectura del Guayas, 2013). En el parque Seminario realizaron un inventario de la fauna existente mediante la observación e identificación de las especies con una guía de campo; las especies de aves transitorias que llegan al parque fueron: Thraupis episcopus, Troglodytes aedum, Dives warsewiczi, Myrmia micrura, Brotogeris pirrhopterus, Crotophaga sulcirrostris, Furnarius leucopus y Columba livia (Cascante, 2016).
El principal problema que enfrenta la avifauna, es la pérdida de hábitat natural debido a la expansión agrícola y urbana, obligando a las aves a desplazarse hacia otros lugares o a adaptarse a las zonas urbanas para lograr su supervivencia (González, 2007; Espinoza, 2016). La riqueza y diversidad de la avifauna en el entorno urbano es afectada directa e indirectamente. Directa, debido a los cambios de procesos ecológicos, hábitat y fuentes de alimentación; Indirectamente las aves son afectadas por los predadores, competidores y patógenos (Gónzalez, 2004). Además, está relacionada a las condiciones ambientales de las áreas verdes tales como el tamaño, estado de la vegetación del suelo, presencia de cuerpos de agua, cantidad y edad de árboles (Berget, 2006).

Entre las variables antrópicas que influyen en la comunidad de aves son: el ruido, la contaminación lumínica, contaminación del aire y la presencia humana. Cianch y Fröhlich (2017) demostraron que los niveles excesivos de ruido conducen a la homogeneización de las comunidades de aves, ya que las especies de aves con las llamadas de baja frecuencia, pueden ser ahogadas por el ruido de los vehículos, ocasionando el desplazamiento de las aves hacia otros sitios, y las aves con las llamadas con alta frecuencia se adapten a un ambiente ruidoso. En cambio, la contaminación lumínica, incrementa la densidad de aves porque afectan su reloj biológico, desarrollando a temprana edad su sistema reproductivo.

La contaminación del aire ocasiona el 
estrés oxidativo en aves, esto es debido por compuestos químicos presentes en ambientes urbanos tales como el hollín y NOx, causando daño a las moléculas como proteínas, lípidos y $\mathrm{ADN}$, conduciendo a la muerte prematura de las aves y por último, la variable antrópica más importante es la presencia humana en los parques urbanos, produce estrés a las aves durante la etapa reproductiva y alteran su dieta nutricional debido que los transeúntes brindan alimentos altos en grasas y azúcares (Isaksson, 2018).

Por lo que es de suma importancia la preservación de los parques urbanos ya que existen árboles y arbustos que ayudan a las aves a encontrar un lugar protegido donde pueden descansar y anidar sin la amenaza de gatos, ratones, perros y otros depredadores locales (Silveira 2011), y también son fuentes de recursos alimenticios (De Almeida y Cándido, 2017). Además, es recomendable que en los parques urbanos exista vegetación nativa, porque ayuda a incrementar la presencia y conservación de aves nativas (McKinney, 2002).

La finalidad de este estudio fue analizar la diversidad biológica de la avifauna en tres parques de Guayaquil: Centenario, Seminario y San Agustín para establecer puntos estratégicos de conservación en el área urbana de Guayaquil. El manejo adecuado de las aves en el entorno urbano, brinda beneficios sociales, ambientales y económicos tales como el control biológico de plagas, polinización y dispersión de semillas, así como también, son considerados indicadores de la calidad ambiental.

\section{Materiales y métodos}

\section{Área de estudio}

La investigación se llevó a cabo en la Provincia del Guayas, cantón Guayaquil; en los parques: Centenario, Seminario y San Agustín. Los tres parques de estudio se encuentra en zona comercial y las edificaciones que rodean a los parques oscilan entre 2-5 pisos.

Las coordenadas del parque Centenario son $2^{\circ} 11^{\prime 2} 23,43$ " S; 79²53'15,64"O y su área es $0,0287 \mathrm{~km}^{2}$. Algunas de las especies arbóreas que se encuentran en el parque son: Samanea saman (Samán), Inga spectabilis (Guaba machete), Guazuma ulmifolia (Guazmo), Psidium guajaba (Guayaba), Ficus elástica (Caucho ornamental), Prosopis juliflora (Algarrobo), Terminalia catappa (Almendro), Mangífera indica (Mango), Acacia nilotica (Aromo), Cedrela odorata (Cedro), Blighia sapida (Aki), Ceiba trichistandra (Ceibo), Spathodea campanulata (Tulipán africano) y, Vitex gigantea (Pechiche).

El área del parque Seminario es 0,0056 $\mathrm{km}^{2}$ y su coordenada geográfica es $2^{\circ} 11$ '41,22" $\mathrm{S} ; 7^{\circ} 52^{\prime} 59,39^{\prime \prime} \mathrm{O}$. Las especies arbóreas que se encuentran en el parque son Ficus microcarpa (Ficus), Ficus obtusifolia (Higuerón), Inga spectabilis (Guaba machete), Cedrela odorata (Cedro), Kigelia africana (Kigelia).

El parque San Agustín está ubicado en las siguientes coordenadas $2^{\circ} 11^{\prime} 16,68^{\prime \prime} \mathrm{S}$; $79^{\circ} 53^{\prime} 15,42^{\prime}$ "O y su área es $0.0011 \mathrm{Km}^{2}$. Este parque consta de cuatro especies arbóreas que son: Samanea Saman (Samán), Mangifera índica (Mango), Ceiba trichistandra 
(Ceibo) y Azadirachta indica (Neem). Las especies arbóreas de los parques de estudio fueron identificadas en el libro de árboles de Guayaquil (Molina, Lavayen y Fabara, 2015).

\section{Monitoreo de aves}

Los días y las horas del monitoreo fueron desde el 15 al 26 de octubre en dos horarios: en la mañana desde las 6:30 a 8:30 a.m. y en la tarde desde las 4:00 a 6:00 p.m. La duración del monitoreo en cada punto de conteo fue de 15 minutos y 2 veces a la semana por cada parque (Ralph et al., 1996; González, 2011). Se lo realizó en este horario porque las aves son más activas debido que dejan sus sitios de refugio para comenzar con la búsqueda de sus alimentos y en el atardecer regresan a sus sitios de refugio.

\section{Puntos de monitoreo de aves}

Se utilizó el método de conteo por puntos; existen dos tipos de conteos por puntos: los conteos extensivos que se los realiza en áreas extensas, que cubre el transecto de carreteras o toda una región; $\mathrm{y}$, los conteos intensivos que se los aplica para áreas de estudio de dimensiones reducidas (Ralph et al., 1996).
En este estudio se utilizó el método de conteos intensivos por tratarse de parques urbanos cuya dimensión no alcanzan las 4 hectáreas. Ralph et al. (1996) recomiendan colocar 9 puntos de conteos en una cuadricula de $3 \times 3$ situados a cada $100 \mathrm{~m}$, lo cual cubre 4 ha. La cantidad de puntos de monitoreo se estimaron de acuerdo con la superficie total de los parques: en el parque Centenario con una superficie total de $28.767 \mathrm{~m}^{2}$ se seleccionó 6 puntos de conteos; en el parque Seminario con una superficie de $5.601 \mathrm{~m}^{2}$ se seleccionó 4 puntos de conteos $y$, en el parque San Agustín con una superficie de $1.187 \mathrm{~m}^{2}$ se seleccionó 2 puntos de conteos (González, 2011; Figura 1; Tabla 1).

Tabla 1.

Puntos de observación de aves en cada uno de los parques urbanos.

\begin{tabular}{|c|c|c|c|}
\hline \multirow{2}{*}{$\begin{array}{c}\text { Nombre del } \\
\text { parque }\end{array}$} & \multirow{2}{*}{$\begin{array}{l}\text { Código de los } \\
\text { puntos de } \\
\text { monitoreo }\end{array}$} & \multicolumn{2}{|c|}{ Coordenadas UTM WSG84 } \\
\hline & & $\mathbf{X}$ & $\mathbf{Y}$ \\
\hline San Agustín & PSA - 01 & 623680 & 9758121 \\
\hline San Agustín & PSA - 02 & 623713 & 9758111 \\
\hline Seminario & PS - 01 & 624216 & 9757345 \\
\hline Seminario & PS - 02 & 624176 & 9757353 \\
\hline Seminario & PS - 03 & 624182 & 9757392 \\
\hline Seminario & PS - 04 & 624217 & 9757382 \\
\hline Seminario & PC - 01 & 623661 & 9757964 \\
\hline Centenario & PC - 02 & 623717 & 9757963 \\
\hline Centenario & PC - 03 & 623737 & 9757922 \\
\hline Centenario & PC - 04 & 623727 & 9757867 \\
\hline Centenario & PC - 05 & 623712 & 9757830 \\
\hline Centenario & PC - 06 & 623642 & 9757851 \\
\hline
\end{tabular}

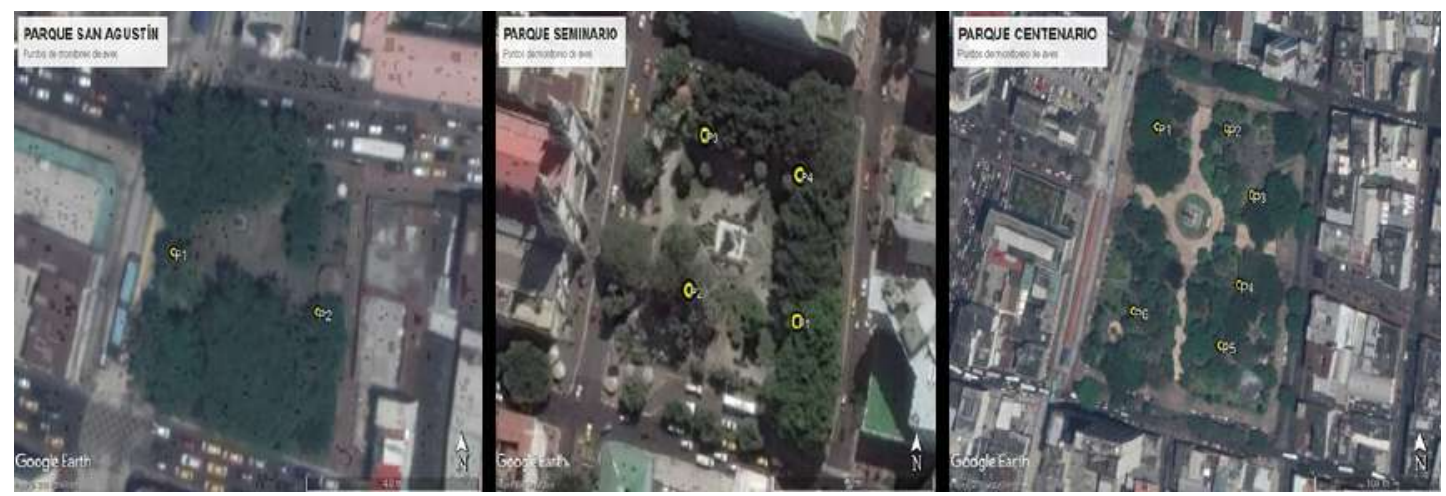

Figura 1. Puntos de monitoreo de aves en los parques San Agustín, Seminario y Centenario.

\section{8}

INVESTIGATIO No. 13, marzo 2020, pp. 25-40, ISSN: 1390 - 6399・ISSN-e: 2602 - 8336 
Durante el monitoreo participaron tres observadores y el esfuerzo que realizaron durante los recorridos de observación por puntos de conteo fue un total de $32 \mathrm{~h}$, considerando 8 días de monitoreo. Las herramientas que se utilizaron fueron los binoculares Baigish $80 \mathrm{X} 80$ y la cámara Nikon D5600 ya que permitió observar las características físicas que presentan las aves, también se utilizó una ficha para el registro de aves. Una vez que se obtuvo el registro de aves se procedió a identificar cada especie utilizando la guía de campo Aves del Ecuador (Ridgely y Greenfield, 2006).

Determinación del uso de hábitat de aves en estratos de vegetación

Para la caracterización del uso de hábitat se analizó la distribución de aves en estratos verticales de vegetación (arbóreo, arbustivo y herbáceo), permitiendo comparar el uso de hábitat común de la avifauna en los parques urbanos Centenario, Seminario y San Agustín, además se consideró en adicionar el uso de hábitat en el espacio abierto y espacio aéreo, clasificándolo de la siguiente manera: 1) Estrato arbóreo: Comprende el dosel o copa de árboles y está cubierto de follaje y su altura es $>3 \mathrm{~m}$, 2) Estrato arbustivo: Es la vegetación $<3 \mathrm{~m}$ y $>0,5 \mathrm{~m}$ de altura de abundante cobertura, 3) Estrato herbáceo: Se caracteriza por la presencia de vegetación inferior a $0,5 \mathrm{~m}$ y por lo general está compuesta por césped y plantas arvenses, 4) Espacio abierto: Es la superficie del suelo y mayormente está constituido por tierra, piedra, cemento o baldosa y, 5) Espacio aéreo: Aves que vuelan sin posarse y se alimentan en el aire ubicándose en la parte alta del área de estudio (González, 2004; Rojas, 2014).

\section{Análisis de Datos}

Para estimar la diversidad de aves en cada uno de los parques se utilizó el índice de Shannon (H'), el cual determina la heterogeneidad de una comunidad sobre la base de dos parámetros que son el número de especies presentes y su abundancia relativa (Ferriol y Merle, 2012; Pla, 2006).

$$
\mathrm{H}=-\sum_{\mathrm{i}=1}^{\mathrm{S}} \mathrm{p}_{\mathrm{i}} \ln \mathrm{p}_{\mathrm{i}}
$$

Donde:

$H=$ diversidad

$S=$ número de especies

$P i=$ proporción del número de individuos de la especie i con respecto al total $(n i / N t)$.

$L n=$ Logaritmo natural

Para la interpretación de valores del índice de Shannon se emplearon los criterios de Jácome (2018) (Tabla 2).

Tabla 2.

Interpretación de valores del índice de Shannon Wienner.

\begin{tabular}{lc}
\hline \multicolumn{2}{c}{ Interpretación de Valores } \\
\hline Diversidad Alta & $3,10-4,50$ \\
Diversidad Media & $1,60-3,00$ \\
Diversidad baja & $0,00-1,50$ \\
\hline
\end{tabular}

\section{Resultados}

En los parques Centenario, Seminario y San Agustín se contabilizó un total de 13 familias de aves y 25 especies (Tabla 3). En el Anexo 1 se ilustran las aves registradas con fotografías. 
Tabla 3.

Especies de aves observadas en los parques Centenario, Seminario y San Agustín

\begin{tabular}{|c|c|c|c|c|c|c|}
\hline No. & Familia & Nombre científico & Nombre común & PSA & PS & PC \\
\hline 1 & COLUMBIDAE & Columba buckleyi & Tortolita ecuatoriana & & $\mathrm{X}$ & $\mathrm{X}$ \\
\hline 2 & COLUMBIDAE & Columba livia & Paloma doméstica & $\mathrm{X}$ & $\mathrm{X}$ & $\mathrm{X}$ \\
\hline 3 & COLUMBIDAE & Zenaida auriculata & Tórtola orejuda & & $\mathrm{X}$ & \\
\hline 4 & COLUMBIDAE & Claravis pretiosa & Tortolita azul & & $\mathrm{X}$ & $\mathrm{X}$ \\
\hline 5 & CUCULIDAE & Crotophaga ani & Garrapatero Piquiliso & $\mathrm{X}$ & $\mathrm{X}$ & $\mathrm{X}$ \\
\hline 6 & EMBERIZIDAE & Sicalis flaveola & Pinzón sabanero azafranado & & $\mathrm{X}$ & \\
\hline 7 & FREGATIDAE & Fregata magnificens & Fragata magnífica & & $\mathrm{X}$ & \\
\hline 8 & FURNARIIDAE & Furnarius cinnamomeus & Hornero del pacífico & & $\mathrm{X}$ & $\mathrm{X}$ \\
\hline 9 & ICTERIDAE & Molothrus oryzivorus & Vaquero gigante & $\mathrm{X}$ & $\mathrm{X}$ & $\mathrm{X}$ \\
\hline 10 & ICTERIDAE & Quiscalus mexicanus & Clarinero coligrande & $\mathrm{X}$ & $\mathrm{X}$ & \\
\hline 11 & MIMIDAE & Mimus longicaudatus & Sisonte coligrande & & $X$ & $\mathrm{X}$ \\
\hline 12 & PICIDAE & Veniliornis kirkii & Carpintero lomirrojo & & $\mathrm{X}$ & $\mathrm{X}$ \\
\hline 13 & POLIOTILIDAE & Polioptila plumbea & Perlita tropical & & & $\mathrm{X}$ \\
\hline 14 & PSITTACIDAE & Aratinga erythrogenys & Perico caretirrojo & $\mathrm{X}$ & $\mathrm{X}$ & $\mathrm{X}$ \\
\hline 15 & PSITTACIDAE & Brotogeris pyrrhopterus & Perico cachetigrís & & $\mathrm{X}$ & $\mathrm{X}$ \\
\hline 16 & PSITTACIDAE & Forpus coelestis & Periquito del pacífico & & $\mathrm{X}$ & \\
\hline 17 & THRAUPIDAE & Euphonia xanthogaster & Eufonia ventrinaranja & & & $\mathrm{X}$ \\
\hline 18 & THRAUPIDAE & Thraupis episcopus & Tangara azuleja & $\mathrm{X}$ & $\mathrm{X}$ & $\mathrm{X}$ \\
\hline 19 & THRAUPIDAE & Ramphocelus nigrogularis & Tangara negricarmesin & & & $\mathrm{X}$ \\
\hline 20 & TROCHILIDAE & Metallura williami & Metalura verde & & & $\mathrm{X}$ \\
\hline 21 & TROCHILIDAE & Damophila julie & Colibrí ventrivioleta & & & $\mathrm{X}$ \\
\hline 22 & TROCHILIDAE & Amazilia amabilis & Amazilia pechiazul & & & $\mathrm{X}$ \\
\hline 23 & TYRANNIDAE & Tolmomyias sulphurescens & Picoancho azufrado & & & $\mathrm{X}$ \\
\hline 24 & TYRANNIDAE & Conopias albovittata & Mosquero aureola & & & $\mathrm{X}$ \\
\hline 25 & TYRANNIDAE & Tyrannus melancholicus & Tirano tropical & $\mathrm{X}$ & $\mathrm{X}$ & $X$ \\
\hline
\end{tabular}

Nota: Parque San Agustín (PSA), Parque Seminario (PS) y Parque Centenario (PC).

En los parques Centenario, Seminario y San Agustín se registró un total de 25 especies pertenecientes a 13 familias, representando el $4,38 \%$ del total de aves registradas en la provincia del Guayas (Prefectura del Guayas, 2013). La familia Columbidae fue la más observada en las tres áreas de estudio, representando el $16 \%$, seguido de las familias Thraupidae, Trochilidae y Tyrannidae que representan el $12 \%$ cada una; la familia Icteridae representa el $8 \%$ y las familias Cuculidae, Emberizidae, Fregatidae, Furnariidae, Mimidae, Picidae y Poliotilidae son representadas con el 4\% cada una (Figura 2).

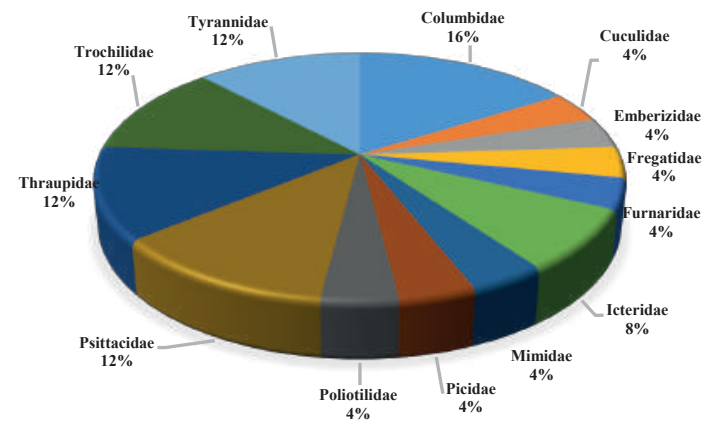

Figura 2. Porcentaje de las familias de aves en los parques Centenario, Seminario y San Agustín.

Estado de conservación de las aves por parques

Los datos sobre el estado de conservación de las aves se compararon 
con la lista roja de la IUCN, e indican que en los tres parques evaluados, aproximadamente el $86 \%$ de las aves observadas se encuentran en la categoría preocupación menor LC; el $11 \%$ se encuentran en la categoría Vulnerable; y, únicamente en el Parque Centenario 3\% de las aves observadas se encuentran en la categoría Casi Amenazada (Figura 3).

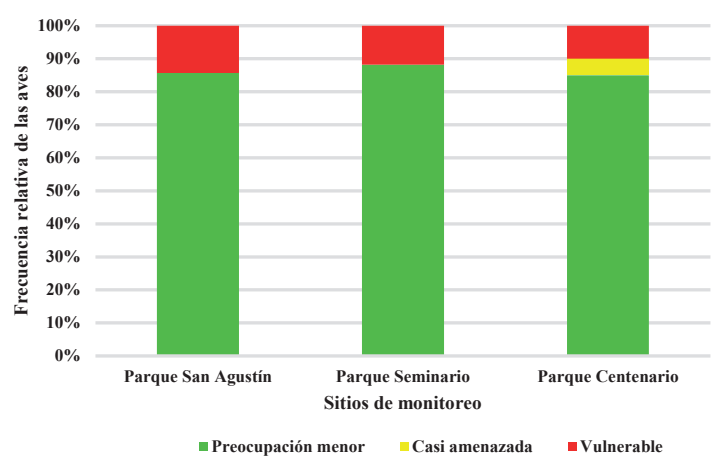

Figura 3. Estado de conservación de las aves en los parques San Agustín, Seminario y Centenario.

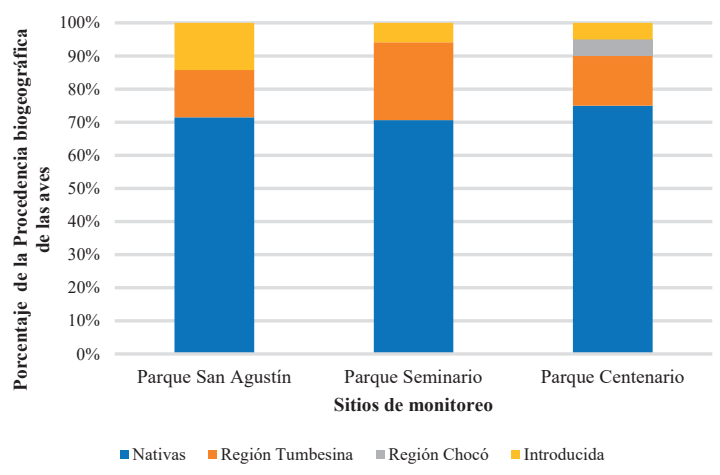

Figura 4. Procedencia biogeográfica de las aves observadas en los parques San Agustín, Seminario y Centenario.

Procedencia biogeográfica de especies de aves por parques

Los datos sobre la procedencia biogeográfica de las aves se obtuvo del listado de aves de la Provincia del Guayas (Prefectura del Guayas, 2013) y los resultados indican que en los parques evaluados, aproximadamente entre el $70-72 \%$ de las aves observadas son nativas, entre el $18-25 \%$ especies de la Región Tumbesina, el 5\% son especies introducidas, y únicamente el Parque Centenario presentó un 5\% de especies provenientes de la región Chocó (Figura 4).

Determinación del uso de hábitat de las aves por estratos de vegetación

Se obtuvo como resultado que el Parque San Agustín aproximadamente el 56\% de las especies de aves se observaron en el estrato arbóreo, el 30\% se observaron en el estrato arbustivo y el $15 \%$ en el espacio abierto, no hubo registro de aves en el estrato herbáceo, ni en el espacio aéreo. En el Parque Seminario se registró el $52 \%$ de aves en el estrato arbóreo, el $18 \%$ en el estrato arbustivo, el $11 \%$ en el estrato herbáceo, el $11 \%$ en el espacio abierto y el $5 \%$ en el espacio aéreo. Por último, en el Parque Centenario se observaron el $74 \%$ de las aves en el estrato arbóreo, el $16 \%$ en el estrato arbustivo y el $10 \%$ en el espacio abierto, no se registraron aves en el estrato arbustivo ni en el espacio aéreo. Se afirma que en los tres parques evaluados están presente todos los estratos: arbóreo, arbustivo, herbáceo, espacio abierto y espacio aéreo (Figura 5).

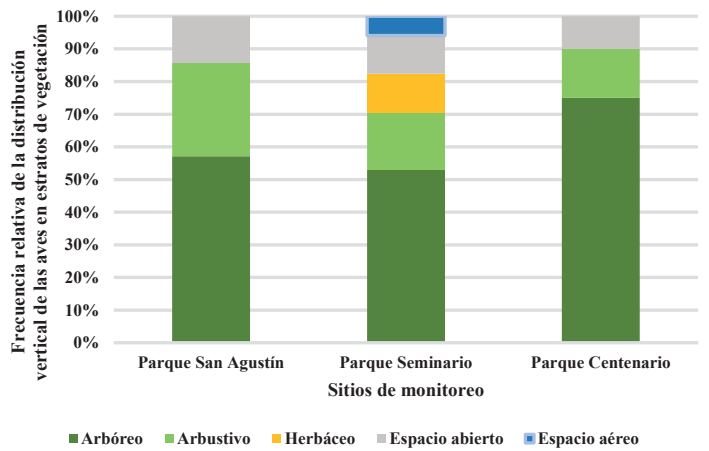

Figura 5. Uso de hábitat de las aves por estratos de vegetación en los parques San Agustín, Seminario y Centenario. 
Se determinó que en la distribución vertical de las aves en estratos de vegetación, más del $50 \%$ de las especies se encontraron en el estrato arbóreo. En los parques San Agustín y Centenario se encontraron especies en el estrato arbóreo $(>50 \%)$, arbustivo $(<20 \%)$ y espacio abierto (10\%), a diferencia del Parque Seminario donde también se encontraron especies en los estratos herbáceo (12\%) y espacio aéreo $(5 \%)$. La presencia de aves en el estrato aéreo en el parque Seminario es por la cercanía del afluente Río Guayas, ya que la especie Fregata magnificens es un ave que sobrevuela regularmente por el Río Guayas.

Determinación de la diversidad de la avifauna en los parques, San Agustín, Seminario y Centenario mediante el indice de Shannon-Wiener.

En el Parque San Agustín se registró un total de 77 individuos, siendo Crotophaga ani la especie más abundante con un total de 32 individuos, seguidamente Columba livia con 26 individuos, Thraupis episcopus 8 individuos, Aratinga erythrogenys 5 individuos, Molothrus oryzivorus 3 individuos, Quiscalus mexicanus 2 individuos y la especie Tyrannus melancholicus con un individuo. Se determinó la diversidad de aves mediante el índice de Shannon que consistió en la relación entre la riqueza y la abundancia, y, se obtuvo como resultado $H^{\prime}=1,42$ indicando que posee una diversidad baja.

En el parque Seminario se registraron un total de 1213 individuos, siendo la especie Columba livia con mayor número de individuos observados (1091 individuos), Thraupis episcopus con 36 individuos, Brotogeris pyrrhopterus con 13 individuos, Columba buckleyi con 11 individuos, Crotophaga ani con 10 individuos, Molothrus oryzivorus con 9 individuos, Tyrannus melancholicus con 8 individuos, Fumarius cinnamomeus con 8 individuos, Fregata magnificens con 5 individuos, Aratinga erythrogenys con 5 individuos, Claravis pretiosa con 5 individuos, Forpus coelestis con 4 individuos, Quiscalus mexicanus con 3 individuos, Sicalis flaveola con 2 individuos y las especies con solo un individuo observadas fueron Mimus longicaudatus, Zenaida auriculata y Veniliornis kirkii. La especie Columba livia es más abundante porque los visitantes del parque le suministran alimentos (balanceado, alpiste, restos de alimentos como panes) causando la aparición de un gran número de individuos de esta especie. En el Parque Seminario se determinó la diversidad de aves mediante el índice de Shannon, y, se obtuvo como resultado $H^{\prime}=0,56$ indicando que posee una diversidad baja.

En el Parque Centenario se contabilizó un total de 573 individuos, la especie más numerosa Columba livia con 200 individuos, seguidamente Thraupis episcopus con 111 individuos, Crotophaga ani con 45 individuos, Brotogeris pyrrhopterus con 36 individuos, Molothrus oryzivorus con 35 individuos, Euphonia xanthogaster con 24 individuos, Polioptila plumbea con 20 individuos, Columba buckleyi con 16 individuos, Tolmomyias sulphurescens con 16 individuos, Tyrannus 
melancholicus con 14 individuos, Furnarius cinnamomeus 13 individuos, Veniliornis kirkii con 13 individuos, Aratinga erythrogenys con 12 individuos, Claravis pretiosa con 6 individuos, Damophila julie con 5 individuos, Conopias albovittata con 2 individuos, Mimus longicaudatus con 2 individuos, Amazilia amabilis con 1 individuo, Metallura williami con 1 individuo y Ramphocelus nigrogularis con 1 individuo. En el Parque Centenario se determinó la diversidad de aves mediante el índice de Shannon y se obtuvo como resultado $H^{\prime}=2,18$ indicando que posee una diversidad media (Tabla 4).

\section{Tabla 4.}

Valores de Riqueza, Abundancia y Diversidad de Shannon en los Parques San Agustín, Seminario y Centenario

\begin{tabular}{lrrrrl}
\hline $\begin{array}{c}\text { Nombre del } \\
\text { parque }\end{array}$ & \multicolumn{2}{c}{ Riqueza Abundancia } & $\begin{array}{c}\text { Diversidad } \\
\text { de Shannon }^{\mathrm{a}}\end{array}$ & $\begin{array}{c}\text { Interpretación } \\
\text { de valores }\end{array}$ \\
\hline San Agustín & 7 & 77 & 1,42 & Diversidad baja \\
Seminario & 17 & 1213 & 0,56 & Diversidad baja \\
Centenario & 20 & 573 & 2,18 & Diversidad media \\
\hline
\end{tabular}

${ }^{a}$ Los cálculos del índice de diversidad de Sahnnon se muestran en los Anexos 2-4.

\section{Discusión}

El resultado sobre la procedencia biogeográfica de las aves, demuestra que la mayoría de especies de aves son nativas en los parques urbanos evaluados, lo que corrobora con los datos de países como Chile (Benito et al., 2019); Argentina (Ramirez et al., 2016). Así como también se realizó a escala mundial una recopilación de datos sobre la procedencia biogeográfica de aves y obtuvieron como resultado que la mayoría de especies de aves urbanas son nativas (Aronson et al., 2014).
En los tres parques urbanos evaluados existe presencia de especies de aves que se encuentra en categorías de amenaza, las cuales pertenecen a la familia de los Psitácidos, estas especies aprovechan los árboles para alimentarse y para descanso, por esa razón, los parques urbanos son de vital importancia para la conservación de aves. Lo que concuerda con Muñoz et al., (2018) lo cual destacan el rol de las áreas verdes para promover la presencia y reproducción de aves en el paisaje urbano.

En cuanto a la distribución vertical de las aves en los estratos de vegetación se determinó que en los parques San Agustín, Seminario y Centenario, más del $50 \%$ de las aves se encuentran en el estrato arbóreo, se argumenta que en el estrato arbóreo hay mayor número de aves debido a la disponibilidad de alimentos por existir árboles fructíferos tales como Mangifera indica (Mango), Vitex gigantea (Pechiche), Inga spectabilis (Guaba machete) y Psidium guajaba (Guayaba); asimismo existen árboles con una gran cobertura vegetal que son utilizados como sitios de refugios tales como Samanea saman (Samán), Guazuma ulmifolia (Guasmo), Ficus obtusifolia (Higuerón), Kigelia africana (árbol de salchichas) y Azadirachta indica (Neem) logrando que las aves tengan los recursos necesarios para su supervivencia en el entorno urbano. Hay que recalcar que la mayoría de especies arbóreas en los parques urbanos de Guayaquil son especies introducidas (Vásquez, 2018). Este resultado concuerda con el estudio de Gómez (2006), quien determinó que la mayor diversidad de aves se encuentra en la 
vegetación arbórea introducida en los parques urbanos. Gallina y Gimenes (2006) demuestran que el estrato que tuvo mayor porcentaje de especies de aves con alta frecuencia de ocurrencia fue el estrato superior o estrato arbóreo. Es importante recalcar que el estrato arbóreo puede influir de manera positiva sobre la riqueza de especies de aves nativas, ya que ellas lo utilizan para nidificar, alimentarse y/o buscar refugio (Díaz y Armesto, 2003).

Las especies Columba livia y Crotophaga ani corresponden a las especies más abundantes en los tres sitios de estudio. Esto puede deberse a la presencia de vegetación exótica en estos parques, vegetación que en otros estudios han estado asociadas al aumento de aves oportunistas o sinantrópicas (Garitano y Gismondi, 2003; Pineda, 2013; Jácome, 2019). En el estudio publicado por Garitano y Gismondi (2003) acerca de la variación de la riqueza y diversidad de la avifauna en 27 áreas verdes urbanas de las ciudades de La Paz y El Alto, Bolivia, menciona que el reemplazo o eliminación de la vegetación natural atrae a especies de aves oportunistas tanto como introducidas o nativas sinantrópicas como la especie Columba livia que fue detectada como dominante en áreas verdes de la zona central de las ciudades. Asimismo, Pineda (2013) menciona que la vegetación presente en los parques urbanos del centro de México está formada en su mayor parte de especies arbóreas exóticas, por lo que la cobertura arbórea fue la principal característica asociada a la presencia de especies de aves exóticas. Jácome (2019), concluyó que el reemplazo o eliminación de la vegetación natural incrementa en las ciudades las poblaciones de aves oportunistas, sean éstas introducidas o nativas sinantrópicas como en el caso de Columba livia, que ha sido registrada en la mayoría de los parques urbanos de la ciudad de Sangolquí, Pichincha.

En la determinación de riqueza y diversidad de aves en los parque urbanos Centenario, Seminario y San Agustín, se puede indicar que el Parque Centenario es donde se concentró la mayor diversidad y riqueza de especies de aves, le sigue el parque Seminario y por último, el parque San Agustín. Este resultado puede deberse a que el Parque Centenario es el parque con mayor superficie de los tres parques estudiados, este parque cuenta con $28.767 \mathrm{~m}^{2}$, el Parque Seminario cuenta con $5.601 \mathrm{~m}^{2}$ y por último, el parque San Agustín cuenta con $1.187 \mathrm{~m}^{2}$. Mayor superficie implica un área más extensa donde existe mayor abundancia de árboles y por ende, disponibilidad de alimentos, sitios de refugio y nidación. Lo que concuerda con Berget (2006), quién analizó la riqueza de aves en 18 parques urbanos de diferentes superficies en Bogotá, Colombia obteniendo como resultado que en los parques con mayor superficie se concentraban mayor riqueza de especies a diferencia de aquellos con menor superficie que albergaban menor número de especies de aves. Asimismo, Muñoz y Norambuena (2018) afirman que en las áreas verdes con mayor extensión hay mayor número de árboles que sirven como fuente de alimentos lo que haría conducir a un aumento de la riqueza de especies de aves. 


\section{Agradecimientos}

Se agradece a la consultora ambiental SAMBITO, SA por facilitar la información de la superficie total de los parques y a Wilson Zambrano por formar parte del equipo de observadores de aves.

\section{Referencias}

Aronson, M., La Sorte, F., Nilon, Ch., Katti, M., Goddard, M., Lepczyk, Ch., Warren, P., Williams, N., Cilliers, S., Clarkson, B., Dobbs, C., Dolan, R., Hedblom, M., Klotz, S., Kooijmans, J., Kühn, I., MacGregor-Fors, I., McDonnell, M., Mörtberg, U., Pysek, P., Siebert, S., Sushinsky, J., Werner, P. y Winter, M. (2014). A global analysis of the impacts of urbanization on bird and plant diversity reveals key anthropogenic drivers. Biological Sciencies, 281, https://doi.org/10.1098/rspb.2013.3330

Benito, J., Escobar, M. y Villaseñor, N. (2019). Conservación en la ciudad: ¿Cómo influye la estructura del hábitat sobre la abundancia de especies de aves en una metrópoli Latinoamericana? Gayana, 83 (1), $114-125$.

Berget, C. (2006). Efecto del tamaño y la cobertura vegetal de parques urbanos en la riqueza y diversidad de la avifauna de Bogotá, Colombia. Gestión y ambiente, 9(2), 45-60.

BirdLife International. (2018). Data Zone. Ecuador: Aves y conservación. Recuperado de: http://datazone.birdlife.org/country/ecuad or/ibas

Burneo, S. (2009). Megadiversidad. Letras Verdes, Revista Latinoamericana de Estudios Socioambientales,10.17141/letrasverdes.3.2 009.822. Recuperado de: https://www.researchgate.net/publication /273187347_Megadiversidad
Cascante, H. (2016). Caracterización del inventario de fauna silvestre presente en el parque Seminario de Guayaquil. Tesis de Pregrado. Universidad Agraria del Ecuador, Guayaquil, Ecuador.

Ciach, M. \& Fröhlich, A. (2017). Habitat type, food resources, noise and light pollution explain the species composition, abundance and stability of a winter bird assemblage in an urban environment. Urban Ecosyst, 20(1), 547 - 559.

De Almeida, A. y Cándido, J. (2017). La importancia de parques urbanos para la conservación de aves. Archivos de Ciencias Veterinarias y Zoología, 2(4), 189 - 199.

Díaz, I. y Armesto, J. (2003). La conservación de las aves silvestres en ambientes urbanos de Santiago. Revista Ambiente $y$ Desarrollo de CIPMA, 19(2), 31- 38. Recuperado de:

https://www.researchgate.net/publication/ 242091660_La_conservacion_de_las_aves silvestres_en_ambientes_urbanos_- de_Sa ntiago

eBird. (2019). Global Big Day. Laboratorio de Ornitología de Cornell, Ithaca, New York. Disponible en: https://ebird.org/globalbigday

Espinoza, W. (2016). Análisis parcial de la diversidad de aves en el bosque y vegetación protectora cerro El Paraíso. Tesis de Maestría. Universidad de Guayaquil, Ecuador. Recuperado de: http://repositorio.ug.edu.ec/bitstream/red ug/13843/1/TTE\%20-\%20WENDY\%20 ESPINOZA\%20pdf.pdf

Ferriol, M. \& Merle, H. (2012). Los componentes alfa, beta y gamma de la biodiversidad. Universidad Politecnica de Valencia. Recuperado de: http://riunet.upv.es/bitstream/handle/102 $51 / 16285 / \mathrm{Micros}$ oft $\% 20$ Word $\% 20$ -\%20articulo\%20docente $\% 20$ def.pdf?seq uence $=1$ 
Freile, J., Brinkhuizen, P., Greenfield, M,. Lysinger, L., Navarrete, J., Nilsson, R., Ridgely, A., Solano-Ugalde, R., Ahlma. \& Boyla, A. (2018). Lista de las aves del Ecuador/Checklist of the Birds of Ecuador. Comité Ecuatoriano de Registros Ornitológicos. Recuperado de: https://ceroecuador.wordpress.com

Gallina, A., Gimenes, M. (2006). Riqueza, composición y distribución espacial de las comunidades de aves en un fragmento forestal urbano en Maringá, Norte del Estado de Paraná, Brasil. Acta Scientiarum. Biological Sciencies, 28 (4), $379-388$.

Garitano-Zavala, A. y Gismondi, P. (2003). Variación de la riqueza y diversidad de la ornitofauna en áreas verdes urbanas de las ciudades de La Paz y El Alto (Bolivia). Ecología en Bolivia, 38(1), 65-78. Recuperado de:

http://www.scielo.org.bo/pdf/reb/v38n1/a 06_v38n1.pdf

Gómez, V. (2006). Aves del parque General San Martín (Mendoza). Distribución y características. Multequina, 16 (1), 81-95.

González, J., Bonache, C., Buzo, D., Díaz, A. y Hernández, L. (2007). Caracterización ecológica de la avifauna de los parques urbanos de la ciudad de Puebla (México). Ardeola, 54(1), 53-67.

González-García, F. (2011). Métodos para contar aves terrestres. En S. Gallina y C. López-González. (Eds.), Manual de Técnicas para el estudio de la Fauna (pp. 85-116). Querétaro, México. Recuperado en: https://www.uaq.mx/FCN/Investigacion/ MANUAL_DE_TECNICAS_PARA_EL _ESTUDIO_DE_LA_FAUNĀ.pdf

González, O. (2004). Ecología de Aves Urbanas en un Parque de la Ciudad de Lima. Tesis de Maestría. Universidad Nacional Mayor de San Marcos. Lima, Perú. Recuperado de: https://www.researchgate.net/profile/Osc ar Gonzalez26/publication/325581908 Ecologia_de_aves_en_un_parque_urban o_de_la_ciudad_de_Lima/links/5b1716e c0f7e-9b1912b37efa/Ecologia-de-aves-en -un-parque-urbano-de-la-ciudad-deLima. pdf?origin=publication_detail

Isaksson C. (2018) Impact of Urbanization on Birds. In: Tietze D. (Ed.), Bird Species (pp.235-257). Cham, Switzerland. Fascinating Life Sciences: Springer.

International Union for Conservation of Nature. (2018). The IUCN Red List of Threatened Species. Available in: http://www.iucnredlist.org

Jácome, I. (2019). Riqueza, Diversidad y abundancia relativa de las aves urbanas de 11 parques de la ciudad de Sangolquí, Cantón Rumiñahui, Ecuador. Siembra, 6(1), 1- 14

Jácome, N. (2018). Estudio de Impacto Ambiental del proyecto "Dragado de la II fase y disposición de los sedimentos de los alrededores del islote El Palmar en la Provincia del Guayas considerando como sitios de depósito "Isabel Ana, lotización "El Tejar", "Caracoles" y terrenos particulares del cantón "Durán". Prefectura del Guayas.

Mclnney, M. (2002). Urbanization, biodiversity and conservation. BioSciencie, 52(1), 883-890.

Ministerio del Ambiente. (2015). Lista de aves del Ecuador. Ecuador: Sistema Único de Información Ambiental. Recuperado de: http://suia.ambiente.gob.ec/documentos? p_p_id $=20 \& p \_p \_l i f e c y c l e=0 \& p \_p \_s t a t e$ $=$ normal $\& p \_p \_$mode $=$view $\& p \_p \_c o l \_i d$ $=$ column $1 \& \mathrm{p} \_$p_col_pos $=1 \& \mathrm{p} \_$_p_col_co unt $=2 \& \_20 \_$struts_action $=\% 2$ Fdocument

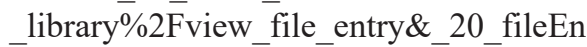
tryId=346666

Molina, N., Lavayen, J y Fabara, M. (2015). Árboles de Guayaquil. En M. Cazorla (Ed). Samborondón: Universidad Espíritu Santo, Ecuador 
Muñoz, A. y Norambuena, H. (2018). Effects of vegetation strata and human disturbance on the diversity of birds in green areas in a city in southern Chile. doi: 10.1186/s40657-018-0130-9

Muñoz, C., Undurraga, M., Saratscheff, T., Rannou, T. y Celis-Diez, J. (2018). Diversidad y conocimiento de las aves urbanas por habitantes de Santiago, Chile. En I. Lazzoni y J. Figueroa (Eds).Biodiversidad urbana en Chile: estado del arte y los desafíos futuros ( p p.283-315). Santiago de Chile: Universidad Central.

Pla, L. (2006). Biodiversidad: Inferencia basada en el índice de Shannon y la riqueza. Interciencia, 31(8), 583-590. Recuperado de: http://www.redalyc.org/pdf/339/3391190 6.pdf

Pineda-López, R., Malagamba A., Arce, 1., y Ojeda, J. (2013). Detección de aves exóticas en parques urbanos del centro de México. Huitzil, 14(1), 56-64. Disponible en: http://www.scielo.org.mx/scielo.php?scri $\mathrm{pt}=$ sci_arttext\&pid=S187074592013000 $100008 \& \operatorname{lng}=$ es\&tlng $=$ es.

Prefectura del Guayas. (2013). Listado de las aves del Guayas. Recuperado de: http://www.guayas.gob.ec/turismo/avituri smo/aves-del-guayas $1 . h t m l$

Ralph, C., Geupel, G., Pyle, P., Martín, T., DeSante, D. y Milá, B. (1996). Manual de métodos de campo para el monitoreo de aves terrestres. California, Estados Unidos: Pacific Southwest Research Station.

Ramírez, C., Marateo, G. y De Beláustegui, H. (2016). Inventario de aves del barrio Centro de la Ciudad de Luján, Buenos Aires, durante el período no reproductivo. Revista del Museo de La Plata, 1 (1), 21-28.

Ridgely, R. \& Greenfield, P. (2006). Aves del Ecuador. Quito, Ecuador: Jocotocco.
Rojas, M. (2014). Diversidad y uso de hábitat de aves en diferentes gradientes urbanos en la ciudad de Guayaquil - Ecuador. Tesis de Pregrado. Universidad de Guayaquil, Guayaquil, Ecuador.

Silveira, L. (2011). Mundo de aves: ¿Qué tal abrir un "restaurante" para aves? Caes y Cia, 390, 64 - 65. Disponible en:

https://www.researchgate.net/publication/ 273369832_Mundo_das_Aves_Que_tal_ abrir_um_restaurante_para_aves

Vásquez, V. (2018). Composición florística de árboles $\mathrm{y}$ arbustos de diez parques urbanos de la ciudad de Guayaquil. Tesis de Pregrado. Universidad de Guayaquil, Guayaquil, Ecuador. 
Anexo 1.

Aves observadas en los parques San Agustín, Seminario y Centenario

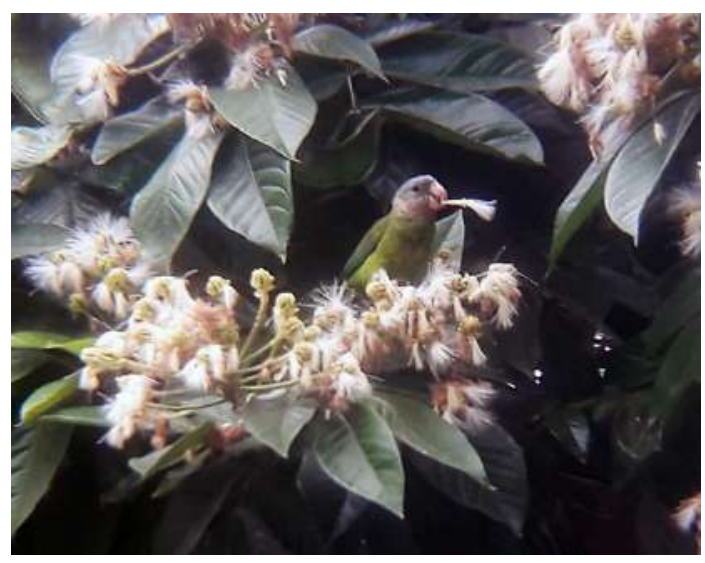

Brotogeris pyrrhopterus

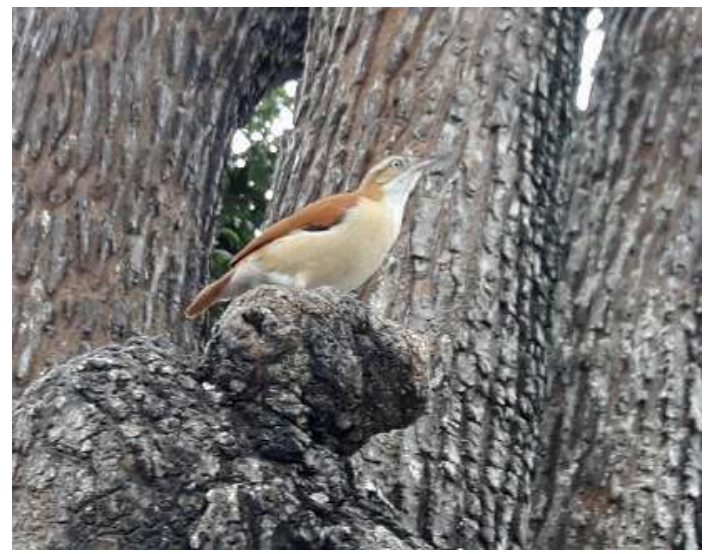

Furnarius cinnamomeus

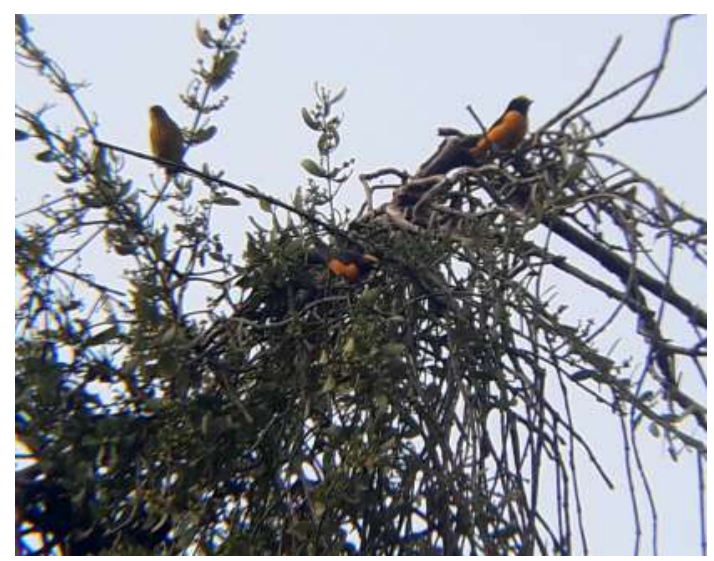

Euphonia xanthogaster

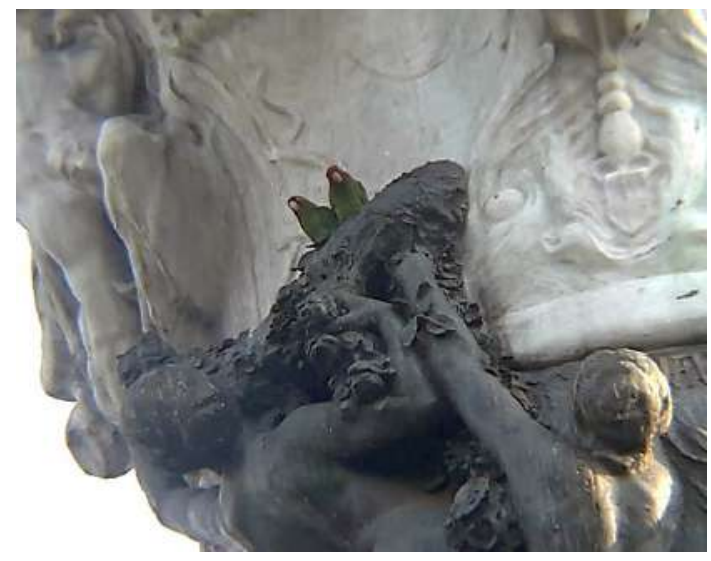

Aratinga erythrogenys

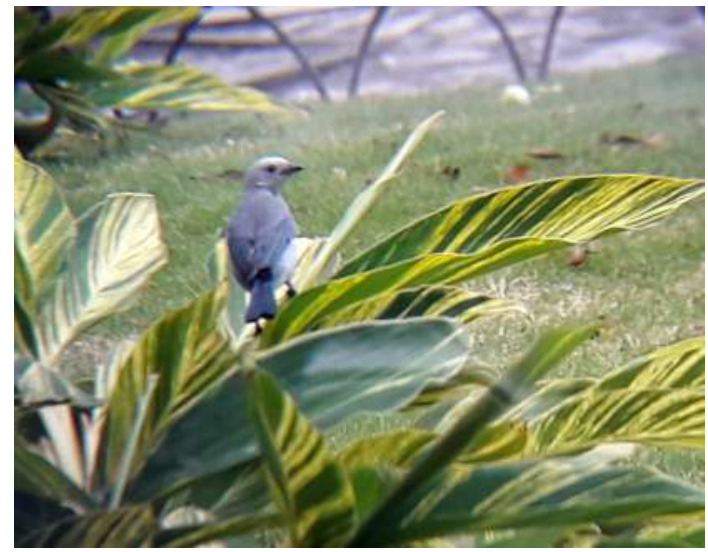

Thraupis episcopus

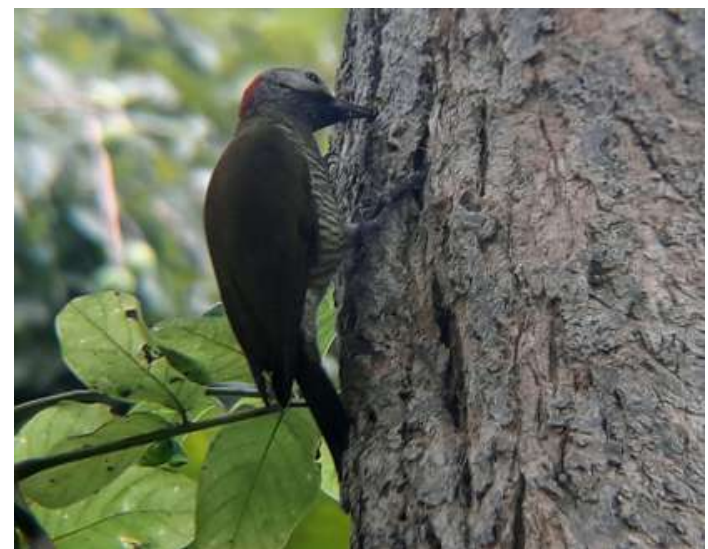

Veniliornis kirkii

38

INVESTIGATIO No. 13, marzo 2020, pp. 25-40,

ISSN: 1390 - 6399・ISSN-e: 2602 - 8336 
Anexo 2.

Índice de Shannon-Wiener para determinar la diversidad de aves en el Parque San Agustín

\begin{tabular}{llcccc}
\hline No. & Especie & Cant. & Abundancia “Pi” & LN “Pi" & Pi* $\mathbf{L N}(\mathbf{P i})$ \\
\hline 1 & Columba livia & 26 & 0,338 & $-1,085709$ & $-0,366603$ \\
2 & Crotophaga ani & 32 & 0,416 & $-0,87807$ & $-0,364912$ \\
3 & Quiscalus mexicanus & 2 & 0,026 & $-3,650658$ & $-0,094822$ \\
4 & Molothrus oryzivorus & 3 & 0,039 & $-3,245193$ & $-0,126436$ \\
5 & Aratinga erythrogenys & 5 & 0,065 & $-2,734368$ & $-0,177556$ \\
6 & Thraupis episcopus & 8 & 0,104 & $-2,264364$ & $-0,235259$ \\
7 & Tyrannus melancholicus & 1 & 0,013 & $-4,343805$ & $-0,056413$ \\
\hline & TOTAL & 77 & & & $-1,422001$ \\
\hline & & & & $\boldsymbol{H}^{\prime}=\mathbf{- 1 , 4 2 2 0 0 1}$
\end{tabular}

Anexo 3.

Índice de Shannon para determinar la diversidad de aves en el parque Seminario

\begin{tabular}{llllll}
\hline No. & Especie & Cant. & Abundancia "Pi” & LN “Pi” & Pi*LN(Pi) \\
\hline 1 & Columba livia & 1091 & 0,8994 & $-0,106002$ & $-0,0953406$ \\
2 & Columba buckleyi & 11 & 0,0091 & $-4,702957$ & $-0,0426484$ \\
3 & Claravis pretiosa & 5 & 0,0041 & $-5,491414$ & $-0,0226357$ \\
4 & Zenaida auriculata & 1 & 0,0008 & $-7,100852$ & $-0,0058540$ \\
5 & Crotophaga ani & 10 & 0,0082 & $-4,798267$ & $-0,0395570$ \\
6 & Sicalis flaveola & 2 & 0,0016 & $-6,407705$ & $-0,0105651$ \\
7 & Fregata magnificens & 5 & 0,0041 & $-5,491414$ & $-0,0226357$ \\
8 & Furnarius cinnamomeus & 8 & 0,0066 & $-5,021410$ & $-0,0331173$ \\
9 & Quiscalus mexicanus & 3 & 0,0025 & $-6,002240$ & $-0,0148448$ \\
10 & Molothrus oryzivorus & 9 & 0,0074 & $-4,903627$ & $-0,0363831$ \\
11 & Mimus longicaudatus & 1 & 0,0008 & $-7,100852$ & $-0,0058540$ \\
& Veniliornis kirkii & 1 & 0,0008 & $-7,100852$ & $-0,0058540$ \\
13 & Forpus coelestis & 4 & 0,0033 & $-5,714558$ & $-0,0188444$ \\
14 & Brotogeris pyrrhopterus & 13 & 0,0107 & $-4,535903$ & $-0,0486123$ \\
15 & Aratinga erythrogenys & 5 & 0,0041 & $-5,491414$ & $-0,0226357$ \\
16 & Thraupis episcopus & 36 & 0,0297 & $-3,517333$ & $-0,1043891$ \\
17 & Tyrannus melancholicus & 8 & 0,0066 & $-5,021410$ & $-0,0331173$ \\
\hline & TOTAL & 1213 & & & $-0,5628882$ \\
\hline
\end{tabular}


Anexo 4.

Índice de Shannon para determinar la diversidad de aves en el parque Centenario

\begin{tabular}{lllccc}
\hline No. & Especie & Cant. & Abundancia “Pi” & LN “Pi" & Pi* $\mathbf{L N}(\mathbf{P i})$ \\
\hline 1 & Columba buckleyi & 16 & 0,02792 & $-3,578297$ & $-0,099918$ \\
2 & Columba livia & 200 & 0,34904 & $-1,052568$ & $-0,367389$ \\
3 & Claravis pretiosa & 6 & 0,01047 & $-4,559126$ & $-0,047740$ \\
4 & Crotophaga ani & 45 & 0,07853 & $-2,544223$ & $-0,199808$ \\
5 & Furnarius cinnamomeus & 13 & 0,02269 & $-3,785936$ & $-0,085894$ \\
6 & Molothrus oryzivorus & 35 & 0,06108 & $-2,795538$ & $-0,170757$ \\
7 & Mimus longicaudatus & 2 & 0,00349 & $-5,657739$ & $-0,019748$ \\
8 & Veniliornis kirkii & 13 & 0,02269 & $-3,785936$ & $-0,085894$ \\
9 & Polioptila plumbea & 20 & 0,03490 & $-3,355153$ & $-0,117108$ \\
10 & Aratinga erythrogenys & 12 & 0,02094 & $-3,865979$ & $-0,080963$ \\
11 & Brotogeris pyrrhopterus & 36 & 0,06283 & $-2,767367$ & $-0,173866$ \\
12 & Euphonia xanthogaster & 24 & 0,04188 & $-3,172832$ & $-0,132893$ \\
13 & Thraupis episcopus & 111 & 0,19372 & $-1,641356$ & $-0,317959$ \\
14 & Ramphocelus nigrogularis & 1 & 0,00175 & $-6,350886$ & $-0,011084$ \\
15 & Metallura williami & 1 & 0,00175 & $-6,350886$ & $-0,011084$ \\
16 & Damophila julie & 5 & 0,00873 & $-4,741448$ & $-0,041374$ \\
17 & Amazilia amabilis & 1 & 0,00175 & $-6,350886$ & $-0,011084$ \\
18 & Tolmomyias sulphurescens & 16 & 0,02792 & $-3,578297$ & $-0,099918$ \\
19 & Conopias albovittata & 2 & 0,00349 & $-5,657739$ & $-0,019748$ \\
20 & Tyrannus melancholicus & 14 & 0,02443 & $-3,711828$ & $-0,090690$ \\
\hline & TOTAL & 573 & & & $-2,184916$ \\
\hline & & & & & $\boldsymbol{H}$ ' $=\mathbf{- 2 , 1 8 4 9 1 6}$ \\
\hline
\end{tabular}

